\title{
Supporting Information for \\ Color tuning in Rhodopsins: the mechanism for the spectral shift between Bacteriorhodopsin and Sensory Rhodopsin II
}

\author{
Michael Hoffmann, ${ }^{1}$ Marius Wanko, ${ }^{1}$ Paul Strodel,${ }^{2}$ Peter H. König, ${ }^{1}$ Thomas Frauenheim, ${ }^{1}$ \\ Klaus Schulten, ${ }^{3}$ Walter Thiel, ${ }^{4}$ Emad Tajkhorshid ${ }^{\dagger,}{ }^{3}$ and Marcus Elstner ${ }^{*, 1,2}$ \\ ${ }^{1}$ Theoretische Physik, Universität Paderborn, Warburger Str. 100, 33098 Paderborn, Germany \\ ${ }^{2}$ Molekulare Biophysik, Deutsches Krebsforschungszentrum, 69120 Heidelberg, Germany \\ ${ }^{3}$ Beckmann Institute, University of Illinois at Urbana-Champaign, \\ 405 North Mathews, Urbana, Illinois 61801, USA \\ ${ }^{4}$ MPI für Kohlenforschung, 45470 Mülheim an der Ruhr, Germany \\ E-mail:* elstner@phys.uni-paderborn.de, †'emad@ks.uiuc.edu
}

\section{DIPOLE MOMENTS AND MULLIKEN CHARGES FOR QM/MM MINIMIZED STRUCTURES}

Table S-1 contains the dipole moments of the chromophore for the QM/MM optimized structures of bR and ppR. To ensure comparability of the dipole moments, the position of the Schiff base nitrogen has been taken as the point of origin.

TABLE S-1: Dipole moments $\mu$ of the chromophore (in Debye) for the QM/MM optimized structures ${ }^{a}$

\begin{tabular}{|c|c|c|c|c|c|c|c|c|}
\hline & \multicolumn{4}{|c|}{$\mathrm{bR}$} & \multicolumn{4}{|c|}{$\mathrm{ppR}$} \\
\hline & $\mu_{x}$ & $\mu_{y}$ & $\mu_{z}$ & $|\mu|$ & $\mu_{x}$ & $\mu_{y}$ & $\mu_{z}$ & $|\mu|$ \\
\hline \multicolumn{9}{|c|}{ SORCI } \\
\hline$\mu_{\mathrm{S}_{0}}{ }^{b}$ & 3.0 & 12.2 & -5.7 & 13.8 & 3.3 & 8.3 & -3.5 & 9.6 \\
\hline$\mu_{\mathrm{S}_{1}}{ }^{b}$ & 3.8 & 20.2 & -11.6 & 23.6 & 4.4 & 15.0 & -7.6 & 17.4 \\
\hline$\mu_{\mathrm{S}_{0}}^{\text {vacuum } c}$ & 4.5 & 18.7 & -6.6 & 20.3 & 5.3 & 18.0 & -5.0 & 19.4 \\
\hline$\mu_{\mathrm{S}_{1}}^{\text {vacuum } c}$ & 5.0 & 26.2 & $\begin{array}{c}-12.9 \\
\text { OM2/ }\end{array}$ & $\begin{array}{l}29.6 \\
\text { MRC }\end{array}$ & $\begin{array}{l}6.2 \\
\mathrm{II}\end{array}$ & 27.3 & -11.2 & 30.1 \\
\hline$\mu_{\mathrm{S}_{0}}{ }^{b}$ & 1.2 & 11.1 & -4.8 & 12.2 & 1.9 & 8.2 & -2.6 & 8.8 \\
\hline$\mu_{\mathrm{S}_{1}}{ }^{b}$ & 2.0 & 20.4 & -12.1 & 23.8 & 2.9 & 15.9 & -7.9 & 18.0 \\
\hline$\mu_{\mathrm{S}_{0}}^{\text {vacuum } c}$ & 3.2 & 19.3 & -6.0 & 20.5 & 4.4 & 19.9 & -4.4 & 20.9 \\
\hline$\mu_{\mathrm{S}_{1}}^{\text {vacuum } c}$ & 3.5 & 27.3 & -13.3 & 30.6 & 5.1 & 29.6 & -11.5 & 32.2 \\
\hline
\end{tabular}

${ }^{c}$ Geometries from QM/MM optimization of the protein. External point charges not included in QM calculations.

Table S-2 and Table S-3 contain the Mulliken charges and corresponding Cartesian coordinates for the QM/MM optimized structures of $\mathrm{bR}$ and ppR. The dipole moment differences calculated based on the Mulliken charges agree well with the exact values (Table S-1).

\section{GEOMETRICAL DATA FOR THE QM/MM MOLECULAR DYNAMIC SIMULATIONS.}

Table S-4 contains the geometrical parameters of the extended HBN for QM/MM molecular dynamic simulations of bR and ppR.

\section{EXCITATION ENERGIES OF BR AND PPR - ADDITIONAL ANALYSIS}

Preceding theoretical studies ${ }^{1,2}$ proposed structural differences in the (extended) HBN to cause the complete spectral shift, e.g. Hayashi et al. ${ }^{1}$ attributed the spectral shift to an increased distance between the Schiff base nitrogen and Asp212 in bR relative to ppR (5.2 $\AA$ in bR, $4.5 \AA$ in ppR). In contrast, our calculations reproduce a major part of the spectral shift only after incorporating the remainder of the protein environment, which red-shifts the excitation energy in bR to a larger extent than in ppR. 
TABLE S-2: Mulliken charges $Q$ of the chromophore for the QM/MM optimized structures ${ }^{a}$

\begin{tabular}{|c|c|c|c|c|c|c|c|c|}
\hline \multirow[b]{2}{*}{ Atom } & \multicolumn{4}{|c|}{$\mathrm{bR}$} & \multicolumn{4}{|c|}{ ppR } \\
\hline & $Q_{\mathrm{S}_{0}}{ }^{b}$ & $Q_{\mathrm{S}_{1}}{ }^{b}$ & $Q_{\mathrm{S}_{0}}^{\text {vacuum }}$ & $Q_{\mathrm{S}_{1}}^{\mathrm{vac}}$ & $Q_{\mathrm{S}_{0}}{ }^{b}$ & $Q_{\mathrm{S}_{1}}{ }^{b}$ & $Q_{\mathrm{S}_{0}}^{\text {vacuum }}$ & $Q_{\mathrm{S}_{1}}^{\text {vacuum }}$ \\
\hline 1 & -0.31736 & -0.31814 & -0.31383 & -0.31558 & -0.32494 & -0.32538 & -0.31655 & -0.31936 \\
\hline 2 & 0.12787 & 0.12756 & 0.13551 & 0.13492 & 0.10859 & 0.10796 & 0.13746 & 0.13754 \\
\hline 3 & 0.12569 & 0.12543 & 0.13617 & 0.13570 & 0.14056 & 0.14038 & 0.13609 & 0.13540 \\
\hline 4 & 0.09154 & 0.09087 & 0.14772 & 0.14738 & 0.09637 & 0.09606 & 0.14880 & 0.14819 \\
\hline 5 & -0.31389 & -0.31439 & -0.32682 & -0.32521 & -0.31091 & -0.31195 & -0.32549 & -0.32281 \\
\hline 6 & 0.16959 & 0.17022 & 0.13343 & 0.13357 & 0.18987 & 0.19082 & 0.13189 & 0.13195 \\
\hline 7 & 0.10945 & 0.10917 & 0.11317 & 0.11348 & 0.09333 & 0.09288 & 0.11600 & 0.11554 \\
\hline 8 & -0.25675 & -0.25760 & -0.23882 & -0.23923 & -0.26330 & -0.26346 & -0.24098 & -0.24198 \\
\hline 9 & 0.13143 & 0.12576 & 0.15293 & 0.14814 & 0.14403 & 0.13945 & 0.15265 & 0.14776 \\
\hline 10 & 0.16116 & 0.16005 & 0.136 & & 0.15924 & 0.15870 & 0.13510 & 85 \\
\hline 11 & -0.03793 & -0.04180 & -0.06211 & 18 & -0.03169 & -0.03869 & -0.06000 & 209 \\
\hline 12 & 48 & 0.11 & 0.1 & & 2000 & 0.11579 & 27 & \\
\hline 13 & 0.22861 & 0.22177 & $0.16 ؟$ & 0.1 & 0.22114 & 0.21571 & 0.17 & 02 \\
\hline 14 & -0.44576 & -0.46530 & -0.45 & 26 & -0.43240 & -0.44714 & -0.4 & 29 \\
\hline 15 & 0.41594 & 0.40521 & 0.32 & 0. & 42285 & 0.41231 & 89 & \\
\hline 16 & 0.13858 & 0.08521 & 0.15 & 0.1 & 15066 & 0.09269 & & \\
\hline 17 & 0.18280 & 0.16147 & 0.20 & 0.1 & 0.17720 & 0.15903 & 0.20 & 32 \\
\hline 18 & -0.23854 & -0.22490 & -0.27572 & -0.2 & -0.20664 & -0.18945 & -0.2 & 50 \\
\hline 19 & 0.22698 & 0.21560 & 0.15504 & 0.14339 & 0.21854 & 0.20989 & 0.15789 & 0.14321 \\
\hline 20 & -0.02962 & -0.06479 & -0.00162 & -0.04313 & -0.04626 & -0.07925 & -0.00271 & -0.04697 \\
\hline 21 & -0.28809 & -0.30142 & -0.29100 & -0.30566 & -0.28307 & -0.29293 & -0.29898 & -0.31118 \\
\hline 22 & 0.18467 & 0.17217 & 0.18340 & 0.17109 & 0.19815 & 0.18804 & 0.18460 & 0.17206 \\
\hline 23 & 0.13711 & 0.13661 & 0.15616 & & 13848 & 0.13821 & 0.15440 & 65 \\
\hline 24 & 0.15863 & 0.15023 & 0.16 & & 64 & 0.1 & $0.1^{\prime}$ & 66 \\
\hline 25 & -0.20226 & -0.18857 & -0.2 & & -0.17774 & -0.15304 & -0. & 337 \\
\hline 26 & 240 & 0.20131 & & & & 861 & & \\
\hline 27 & -0 & -0.11 & -0.0 & & 86 & -0.13829 & & \\
\hline 28 & 5 & 0.17066 & 0.1 & & & 0.1 & & \\
\hline 29 & -0.21551 & -0.17913 & -0.2 & & -0 & -0.14 & -0. & \\
\hline 30 & 4 & 0.17028 & 0.1 & & & 0.1 & & \\
\hline 31 & -0.06328 & -0.06913 & -0.0 & -0 . & -0.0 & -0.0 & $-0 .($ & 72 \\
\hline 32 & -0.29463 & -0.29180 & -0.2 & -0 . & -0.29448 & -0.28779 & -0.2 & 560 \\
\hline 33 & 0.17630 & 0.17687 & 0.17 & 0.1 & 0.15441 & 0.15787 & 78 & 303 \\
\hline 34 & 0.15135 & 0.15698 & 0.15632 & 0.16052 & 0.13337 & 0.13591 & 0.16906 & 0.16439 \\
\hline 35 & 0.15283 & 0.15445 & 0.16569 & 0.16406 & 0.18469 & 0.18724 & 0.17018 & 0.16607 \\
\hline 36 & -0.20985 & -0.18730 & -0.20753 & -0.16914 & -0.17913 & -0.15854 & -0.19984 & -0.16290 \\
\hline 37 & 0.17209 & 0.18419 & 0.17776 & 0.1 & 0.16 ? & 0.17688 & 0.1 & 44 \\
\hline 38 & -0.18227 & -0.15918 & -0.13514 & -0.1 & -0.19925 & -0.19014 & -0.12974 & -0.12208 \\
\hline 39 & 0.15672 & 0.16869 & 0.16329 & 8 & 0.15727 & 0.16580 & 0.16045 & 87 \\
\hline 40 & 1664 & 0.02748 & 0 & & 4 & 93 & 0. & \\
\hline 41 & 5 & -0.1 & & & & -0 . & & \\
\hline 42 & & -0.30 & -0.2 & & & -0.2 & -0 & 78 \\
\hline 43 & 3 & 0.14 & 0. & & & 0.1 & & \\
\hline 44 & 0 & 0.18 & 0 & & & & & \\
\hline 45 & 0 & 0.1 & & & 5 & 0.1 & & \\
\hline 46 & -0.16538 & -0.15 & -0.1 & & 89 & -0.1 & -0 & 29 \\
\hline 47 & 0.14656 & 0.15350 & $0.1:$ & & 72 & 0.14212 & 69 & 10 \\
\hline 48 & 0.13404 & 0.13878 & 0.15254 & 0.15912 & 0.12925 & 0.13195 & 0.15404 & 0.16156 \\
\hline 49 & -0.21299 & -0.21081 & -0.20864 & -0.20479 & -0.20859 & -0.20654 & -0.21172 & -0.20778 \\
\hline 50 & 0.11572 & 0.11837 & 0.14718 & 0.14996 & 0.12445 & 0.12623 & 0.14592 & 0.14979 \\
\hline 51 & 0.14550 & 0.14611 & 0.13776 & 0.138 & 0.13512 & 0.13513 & 0.13789 & 0.13831 \\
\hline 52 & -0.19006 & -0.18912 & -0.18088 & -0.18041 & -0.17495 & -0.17384 & -0.17415 & -0.17474 \\
\hline 53 & 0.10983 & 0.11045 & 0.13032 & 0.13114 & 0.11797 & 0.11814 & 0.13176 & 0.13263 \\
\hline 54 & 0.18062 & 0.18314 & 0.14108 & 14452 & 0.15235 & 0.15370 & 0.14086 & 0.14544 \\
\hline 55 & -0.39475 & -0.38810 & -0.41468 & .40665 & 40151 & -0.39322 & -0.42004 & -0.41419 \\
\hline 56 & -0 & -0.26442 & -0.2 & 305 & -0.29102 & -0.28935 & -0.26160 & -0.25645 \\
\hline 57 & 0.15463 & 0.15514 & 0.14321 & 0.14416 & 0.15933 & 0.15946 & 0.14017 & 0.14089 \\
\hline 58 & 0.12199 & 0.12322 & 0.13179 & 0.13331 & 0.15155 & 0.15159 & 0.13657 & 0.13869 \\
\hline 59 & 0.11899 & 0.12124 & 0.14012 & 0.14267 & 0.11550 & 0.11682 & 0.13669 & 0.14048 \\
\hline 60 & -0.24911 & -0.24715 & -0.23948 & -0.23520 & -0.23946 & -0.23904 & -0.24556 & -0.24314 \\
\hline 61 & 0.13709 & 0.13738 & 0.13089 & 0.13143 & 0.13333 & 0.13380 & 0.13754 & 0.13827 \\
\hline 62 & 0.12501 & 0.12505 & 0.13528 & & 11982 & 0.12014 & 0.13527 & 0.13662 \\
\hline 63 & 0.12914 & 0.13105 & 0.13662 & 0.13887 & 0.12867 & 0.13017 & 0.13486 & 0.13867 \\
\hline
\end{tabular}

${ }^{a}$ From SORCI calculations on the chromophore. ${ }^{b}$ External point charges included in QM calculations. ${ }^{c}$ Geometries from QM/MM optimization of the protein. External point charges not included in QM calculations. 
TABLE S-3: Cartesian coordinates (in $\AA$ ) of the chromophore for the QM/MM optimized structures ${ }^{a}$

\begin{tabular}{|c|c|c|c|c|c|c|c|}
\hline \multirow[b]{2}{*}{ Atom } & \multirow[b]{2}{*}{ Туре } & \multicolumn{3}{|c|}{$\mathrm{bR}$} & \multicolumn{3}{|c|}{ ppR } \\
\hline & & $\mathrm{x}$ & $\mathrm{y}$ & $\mathrm{z}$ & $\mathrm{x}$ & y & $\mathrm{z}$ \\
\hline 1 & $\mathrm{C}$ & 1.57673 & -2.56265 & 3.44525 & 1.78222 & -2.37887 & 3.41095 \\
\hline 2 & $\mathrm{H}$ & 0.80005 & -3.14199 & 3.96723 & 1.01023 & -2.94773 & 3.95329 \\
\hline 3 & $\mathrm{H}$ & 2.16074 & -3.26835 & 2.83908 & 2.30877 & -3.08606 & 2.75746 \\
\hline 4 & $\mathrm{H}$ & 2.25090 & -2.09896 & 4.17956 & 2.50610 & -1.96106 & 4.12515 \\
\hline 5 & $\mathrm{C}$ & 0.93289 & -1.52157 & 2.56280 & 1.13265 & -1.29325 & 2.58617 \\
\hline 6 & $\mathrm{H}$ & 1.68796 & -1.04533 & 1.91516 & 1.87533 & -0.82274 & 1.92075 \\
\hline 7 & $\mathrm{H}$ & 0.52789 & -0.71290 & 3.20305 & 0.77220 & -0.49215 & 3.26362 \\
\hline 8 & $\mathrm{C}$ & -0.18547 & -2.07743 & 1.70916 & -0.03314 & -1.81479 & 1.77433 \\
\hline 9 & $\mathrm{H}$ & -0.83335 & -2.74489 & 2.30437 & -0.66839 & -2.46955 & 2.39493 \\
\hline 10 & $\mathrm{H}$ & 0.21294 & -2.67566 & 0.87568 & 0.31881 & -2.42148 & 0.92734 \\
\hline 11 & $\mathrm{C}$ & -1.02930 & -0.94063 & 1.16284 & -0.88246 & -0.67202 & 1.25246 \\
\hline 12 & $\mathrm{H}$ & -1.38802 & -0.30349 & 1.99357 & -1.16097 & 0.00558 & 2.08491 \\
\hline 13 & $\mathrm{H}$ & -1.90657 & -1.33094 & 0.61772 & -1.81198 & -1.05552 & 0.79809 \\
\hline 14 & $\mathrm{~N}$ & -0.27847 & -0.11625 & 0.25547 & -0.18819 & 0.08989 & 0.24755 \\
\hline 15 & $\mathrm{H}$ & -0.17750 & -0.49713 & -0.69886 & -0.18027 & -0.32242 & -0.70056 \\
\hline 16 & $\mathrm{C}$ & 0.22291 & 1.06337 & 0.56835 & 0.39647 & 1.24090 & 0.49302 \\
\hline 17 & $\mathrm{H}$ & 0.12871 & 1.38783 & 1.61983 & 0.39644 & 1.57878 & 1.54462 \\
\hline 18 & $\mathrm{C}$ & 0.69898 & 1.92324 & -0.43706 & 0.86799 & 2.07692 & -0.54210 \\
\hline 19 & $\mathrm{H}$ & 0.57888 & 1.57908 & -1.47372 & 0.68367 & 1.75767 & -1.57725 \\
\hline 20 & $\mathrm{C}$ & 1.11428 & 3.24264 & -0.24542 & 1.41602 & 3.33820 & -0.32832 \\
\hline 21 & $\mathrm{C}$ & 1.61969 & 3.73884 & 1.05975 & 1.95039 & 3.76489 & 0.99018 \\
\hline 22 & $\mathrm{H}$ & 2.70103 & 3.92059 & 0.97845 & 3.04608 & 3.82159 & 0.92594 \\
\hline 23 & $\mathrm{H}$ & 1.46780 & 3.01117 & 1.86461 & 1.70864 & 3.05800 & 1.79113 \\
\hline 24 & $\mathrm{H}$ & 1.15733 & 4.69024 & 1.35195 & 1.58747 & 4.75963 & 1.28018 \\
\hline 25 & $\mathrm{C}$ & 1.01420 & 4.11077 & -1.36872 & 1.44432 & 4.24394 & -1.43505 \\
\hline 26 & $\mathrm{H}$ & 0.63980 & 3.66206 & -2.30095 & 1.06896 & 3.86679 & -2.39775 \\
\hline 27 & $\mathrm{C}$ & 1.26136 & 5.46834 & -1.35820 & 1.82900 & 5.56134 & -1.34413 \\
\hline 28 & $\mathrm{H}$ & 1.67448 & 5.95108 & -0.45947 & 2.21994 & 5.95286 & -0.39314 \\
\hline 29 & $\mathrm{C}$ & 0.96925 & 6.24226 & -2.51092 & 1.69938 & 6.43662 & -2.45870 \\
\hline 30 & $\mathrm{H}$ & 0.48894 & 5.70691 & -3.34520 & 1.26707 & 6.00181 & -3.37366 \\
\hline 31 & $\mathrm{C}$ & 1.29516 & 7.57226 & -2.71731 & 2.05471 & 7.77095 & -2.49561 \\
\hline 32 & $\mathrm{C}$ & 2.03053 & 8.38083 & -1.70415 & 2.67683 & 8.48499 & -1.34669 \\
\hline 33 & $\mathrm{H}$ & 3.10077 & 8.42996 & -1.95155 & 2.82930 & 7.82262 & -0.48889 \\
\hline 34 & $\mathrm{H}$ & 1.94189 & 7.94962 & -0.70067 & 2.04551 & 9.32550 & -1.01980 \\
\hline 35 & $\mathrm{H}$ & 1.65906 & 9.41236 & -1.66793 & 3.64966 & 8.90497 & -1.63192 \\
\hline 36 & $\mathrm{C}$ & 0.98606 & 8.12354 & -4.01058 & 1.79424 & 8.49055 & -3.71681 \\
\hline 37 & $\mathrm{H}$ & 0.37093 & 7.48013 & -4.65273 & 1.27491 & 7.90069 & -4.48168 \\
\hline 38 & $\mathrm{C}$ & 32 & 9.32025 & -4.4 & 81 & 9.79726 & -3.93584 \\
\hline 39 & $\mathrm{H}$ & 2.05074 & 9.91052 & -3.74188 & 2.64162 & 10.32105 & -3.11151 \\
\hline 40 & $\mathrm{C}$ & 96 & 9.88411 & -5.79656 & 23 & 10.60213 & -5.11077 \\
\hline 41 & $\mathrm{C}$ & 1.6 & 11.20752 & -5.98 & 2.10 & 11.94819 & -5.06372 \\
\hline 42 & $\mathrm{C}$ & 2.0 & 12.11437 & -4.86 & 2.78 & 12.59418 & -3.89548 \\
\hline 43 & $\mathrm{H}$ & 2.63889 & 11.61779 & -4.07297 & 3.71 & 12.08419 & -3.61699 \\
\hline 44 & $\mathrm{H}$ & 1.17261 & 12.55504 & -4.40416 & 2.14481 & 12.59718 & -3.00284 \\
\hline 45 & $\mathrm{H}$ & 2.67954 & 12.93978 & -5.24880 & 3.03379 & 13.63901 & -4.12143 \\
\hline 46 & $\mathrm{C}$ & 1.60396 & 11.88989 & -7.31008 & 1.71424 & 12.89430 & -6.15615 \\
\hline 47 & $\mathrm{H}$ & 2.62466 & 11.92931 & -7.74457 & 2.61238 & 13.17797 & -6.74546 \\
\hline 48 & $\mathrm{H}$ & 1.31369 & 12.94564 & -7.16492 & 1.35403 & 13.83764 & -5.70547 \\
\hline 49 & $\mathrm{C}$ & 0.68070 & 11.18812 & -8.27412 & 0.66728 & 12.31066 & -7.07309 \\
\hline 50 & $\mathrm{H}$ & 0.76353 & 11.63544 & -9.28233 & 0.50974 & 12.97616 & -7.94139 \\
\hline 51 & $\mathrm{H}$ & -0.37094 & 11.30511 & -7.96241 & -0.30340 & 12.24168 & -6.54700 \\
\hline 52 & $\mathrm{C}$ & 1.05740 & 9.73269 & -8.31256 & 1.12779 & 10.94618 & -7.52274 \\
\hline 53 & $\mathrm{H}$ & 2.12346 & 9.64912 & -8.59834 & 2.11981 & 11.04420 & -8.00317 \\
\hline 54 & $\mathrm{H}$ & 0.47903 & 9.20636 & -9.08915 & 0.45212 & 10.53547 & -8.29414 \\
\hline 55 & $\mathrm{C}$ & 0.83683 & 9.03289 & -6.96411 & 1.21829 & 9.95633 & -6.34908 \\
\hline 56 & $\mathrm{C}$ & -0.66793 & 8.80570 & -6.78234 & -0.20090 & 9.47687 & -6.02789 \\
\hline 57 & $\mathrm{H}$ & -1.17785 & 9.74876 & -6.55168 & -0.85111 & 10.32882 & -5.79487 \\
\hline 58 & $\mathrm{H}$ & -0.89241 & 8.09844 & -5.97526 & -0.24137 & 8.79752 & -5.17148 \\
\hline 59 & $\mathrm{H}$ & -1.10218 & 8.40658 & -7.70708 & -0.62912 & 8.94891 & -6.89179 \\
\hline 60 & $\mathrm{C}$ & 1.57971 & 7.69932 & -7.06604 & 2.09461 & 8.79842 & -6.83476 \\
\hline 61 & $\mathrm{H}$ & 1.47498 & 7.07078 & -6.17618 & 2.26830 & 8.03047 & -6.07562 \\
\hline 62 & $\mathrm{H}$ & 2.65158 & 7.86905 & -7.23243 & 3.07612 & 9.16893 & -7.15699 \\
\hline 63 & $\mathrm{H}$ & 1.19822 & 7.12574 & -7.91973 & 1.62047 & 8.30957 & -7.69241 \\
\hline
\end{tabular}


TABLE S-4: Selected geometrical parameters and their fluctuations of the retinal binding site for QM/MM molecular dynamic simulations ${ }^{a}$. (Distances in $\AA$; the two residue numbers specified refer to bR and ppR, respectively; fluctuations in parentheses)

\begin{tabular}{clcc}
\hline & bR & ppR \\
\hline \hline RET $_{\mathrm{N}_{\zeta}}$ & $\mathrm{O}(\mathrm{W} 402 / 402)$ & $2.88(0.12)$ & $2.87(0.13)$ \\
$\mathrm{O}(\mathrm{W} 402 / 402)$ & $\mathrm{O}_{\delta 2}($ Asp85/75) & $2.62(0.10)$ & $2.63(0.10)$ \\
$\mathrm{O}(\mathrm{W} 402 / 402)$ & $\mathrm{O}_{\delta 2}($ Asp212/201) & $2.90(0.23)$ & $2.79(0.16)$ \\
$\mathrm{RET}_{\mathrm{N}_{\zeta}}$ & $\mathrm{O}_{\delta 1}($ Asp85/75) & $3.81(0.29)$ & $3.73(0.32)$ \\
$\mathrm{RET}_{\mathrm{N}_{\zeta}}$ & $\mathrm{O}_{\delta 2}($ Asp85/75) & $4.31(0.30)$ & $4.26(0.35)$ \\
$\mathrm{RET}_{\mathrm{N}_{\zeta}}$ & $\mathrm{C}_{\gamma}($ Asp85/75) & $4.43(0.24)$ & $4.38(0.29)$ \\
$\mathrm{RET}_{\mathrm{N}_{\zeta}}$ & $\mathrm{O}_{\delta 1}($ Asp212/201) & $4.07(0.32)$ & $4.43(0.32)$ \\
$\mathrm{RET}_{\mathrm{N}_{\zeta}}$ & $\mathrm{O}_{\delta 2}($ Asp212/201) & $4.88(0.26)$ & $4.79(0.26)$ \\
$\mathrm{RET}_{\mathrm{N}_{\zeta}}$ & $\mathrm{C}_{\gamma}($ Asp212/201) & $4.37(0.23)$ & $4.50(0.25)$ \\
$\mathrm{O}(\mathrm{W} 402 / 402)$ & $\mathrm{O}_{\delta 1}($ Asp85/75) & $3.70(0.27)$ & $3.65(0.26)$ \\
$\mathrm{O}(\mathrm{W} 402 / 402)$ & $\mathrm{O}_{\delta 1}($ Asp212/201) & $3.53(0.25)$ & $3.79(0.26)$ \\
$\mathrm{O}(\mathrm{W} 401 / 401)$ & $\mathrm{O}_{\delta 2}($ Asp85/75) & $2.65(0.11)$ & $2.63(0.10)$ \\
$\mathrm{O}(\mathrm{W} 401 / 401)$ & $\mathrm{O}_{(\mathrm{W} 406 / 400)}$ & $2.76(0.13)$ & $2.80(0.15)$ \\
$\mathrm{O}(\mathrm{W} 406 / 400)$ & $\mathrm{O}_{\delta 2}($ Asp212/212) & $2.64(0.10)$ & $2.75(0.25)$ \\
$\mathrm{O}(\mathrm{W} 406 / 400)$ & $\mathrm{N}_{\omega / \delta}($ Arg82/72) & $2.80(0.11)$ & $3.04(0.25)$ \\
$\mathrm{RET}_{\mathrm{N}_{\zeta}}$ & $\mathrm{C}_{\zeta}($ Arg82/72) & $10.42(0.23)$ & $10.33(0.28)$ \\
$\mathrm{RET}_{\mathrm{C} 6}$ & $\mathrm{C}_{\zeta}($ Arg82/72) & $11.72(0.24)$ & $10.95(0.30)$ \\
\hline$a$ &
\end{tabular}

In order to elucidate the differences between the models and the interpretation of the results further, it is helpful to analyze the electrostatic interactions between the chromophore and various parts of the protein environment.

The energy of the QM/MM system for a given electronic state and geometry contains three contributions that may change upon excitation $\left(\right.$ see also $\left.{ }^{1}\right)$ : (1) the energy $E^{0}$ of the isolated chromophore, (2) the electrostatic (Coulomb) interaction energy $V_{\mathrm{ch}-\mathrm{Er}}^{\mathrm{ES}}$ between the polarized chromophore density and the protein, and (3) the electronic reorganization energy $E^{\mathrm{ERO}}$ which is needed to polarize the chromophore and is positive because of the variational principle. The interaction energy $V_{\mathrm{ch}-\mathrm{Er}}^{\mathrm{ES}}$ can be further divided into the interaction of the protein with the unpolarized chromophore $\left(V_{\text {ch-pr }}^{\mathrm{ES}, \mathrm{vac}}\right)$ and the change in $V_{\mathrm{ch}-\mathrm{pr}}^{\mathrm{ES}}$ due to the chromophore polarization $\left(V_{\mathrm{ch}-\mathrm{pr}}^{\mathrm{ES}, \mathrm{p}}\right)$. The total energy gain due to polarization of the chromophore is the sum of $V_{\mathrm{ch} \text {-pr }}^{\mathrm{ES} \text {. }}$ and $E^{\mathrm{ERO}}$.

According to our calculations, both terms $\left(V_{\text {ch-pr }}^{\mathrm{ES}}, E^{\mathrm{ERO}}\right)$ contribute to the shift of ppR vs. bR to the same degree $\left(0.13 \mathrm{eV} \Delta_{\mathrm{ppR}-\mathrm{bR}} E^{\mathrm{ERO}} ; 0.12 \mathrm{eV} \Delta_{\mathrm{ppR}-\mathrm{bR}} V_{\mathrm{ch}-\mathrm{pr}}^{\mathrm{ES}}\right)$. Thus, we investigated the contributions of individual residues to the excitation energy.

The best way to obtain these is by perturbational analysis which requires the calculation of the excitation energy by switching off the partial charges of every side chain successively, as presented in section III C in our article. As a faster alternative, the comparison of $V_{\mathrm{ch}-\mathrm{pr}}^{\mathrm{ES}}$ and $V_{\mathrm{ch}-\mathrm{pr}}^{\mathrm{ES} \text {,va }}$ can be used to estimate the change in $E^{\mathrm{ERO}}$ that is caused by the perturbation from the charges of the individual amino acids. Therefore we assume that approximately $\frac{1}{2} V^{\mathrm{ES}, \text { pol }}$ is invested in the electronic reorganization ${ }^{4}$, i.e., $E^{\mathrm{ERO}} \approx-\frac{1}{2} V_{\mathrm{ch}-\mathrm{pr}}^{\mathrm{ES}, \mathrm{pol}}=-\frac{1}{2}\left(V_{\mathrm{ch}-\mathrm{pr}}^{\mathrm{ES}}-V_{\text {ch-pr }}^{\mathrm{ES}, \mathrm{vac}}\right)$. Hence, we only need the ground-state and excited-state charges from two calculations, one including and one omitting the entire protein charges, to obtain the contributions of individual residues to the total or excitation energy ${ }^{5}$ :

$$
\begin{aligned}
\Delta_{S_{1}-S_{0}} E & =\Delta_{S_{1}-S_{0}} E^{0}+\Delta_{S_{1}-S_{0}} V_{\mathrm{ch}-\mathrm{pr}}^{\mathrm{ES}}+\Delta_{S_{1}-S_{0}} E^{\mathrm{ERO}} \\
& \approx \Delta_{S_{1}-S_{0}} E^{0}+\frac{1}{2} \Delta_{S_{1}-S_{0}} V_{\mathrm{ch}-\mathrm{pr}}^{\mathrm{ES}}+\frac{1}{2} \Delta_{S_{1}-S_{0}} V_{\mathrm{ch}-\mathrm{pr}}^{\mathrm{ES}, \mathrm{pac}}
\end{aligned}
$$

The results for bR and ppR are shown in Fig. 1. The largest contributions to the shift come from the difference between Glu194/Asp193, Ser141/Gly130 and Ala215/Thr204. Although the primary counterion residues Asp85/75 and Asp212/201 dominate the blue shift induced by the protein in both cases, their contribution to the shift of ppR vs. bR is rather small, even negative $(-0.025 \mathrm{eV})$ for the latter residue.

The results for the counterions residues imply that the counterion complex would not affect the ppR-bR shift at all which is in clear contrast to the results obtained with the HBN model (sec. III B) and points out the limitations of an analysis based on eq. (2).

Interestingly, we obtain qualitatively the same results when using Hayashi's QM/MM optimized structures of bR and $\mathrm{ppR}^{1}$. In particular, the counterion complex contributes only very slightly $(0.02 \mathrm{eV})$ to the shift. 

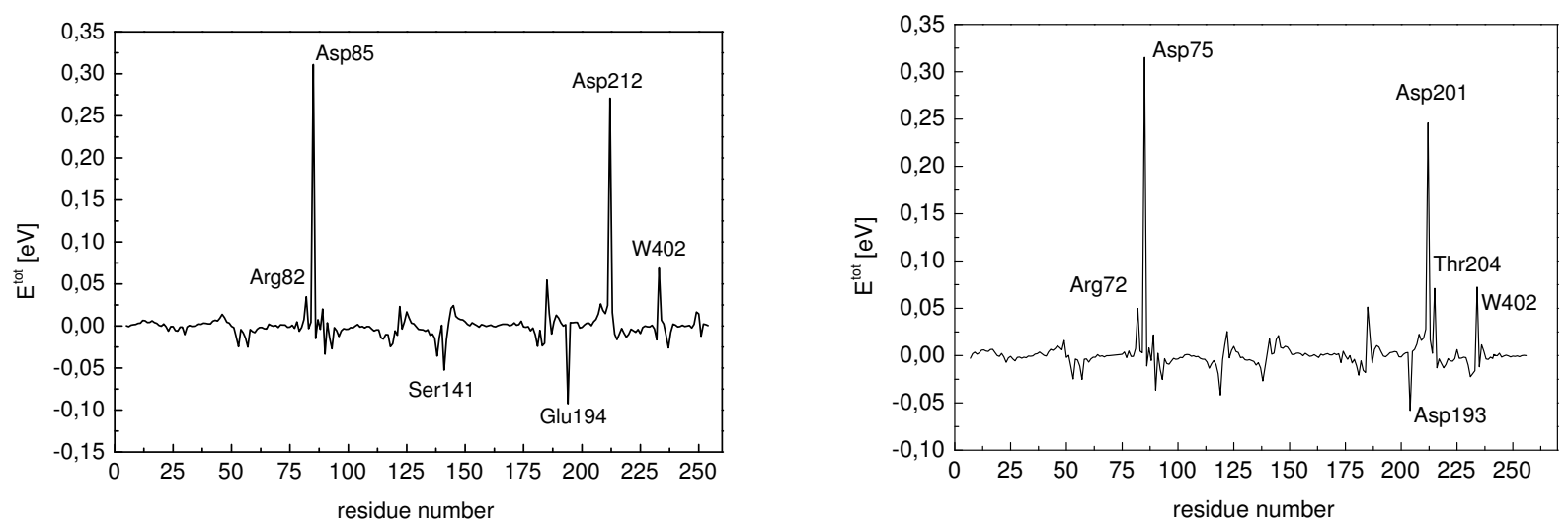

FIG. 1: Calculated contributions to the excitation energy $\Delta_{\mathrm{S}_{1}-\mathrm{S}_{0}}$ E for individual residues for bR (top) and ppR (bottom) based on eq. (2)

In order to investigate the influence of the differences in the geometry of the HBN in the model of Hayashi et al. ${ }^{1}$, as outlined in sec. III A, further, we recalculated the excitation energies using Hayashi's QM/MM optimized structures. In an analogous manner as for the analysis of the crystal structures, only the chromophore geometry was allowed to relax while the position of the Schiff base nitrogen and the rest of the protein was fixed, thus preserving the differences of the HBN.

The calculated excitation energies and the spectral shift (Table S-5) are similar to those evaluated for our QM/MM optimized and the x-ray structures for the whole protein as well as for the HBN model (Table II in sec. III B).

TABLE S-5: Vertical OM2/MRCI excitation energies $\Delta \mathrm{E}_{\mathrm{S}_{1}-\mathrm{S}_{0}}$ (in eV) for previous theoretical structures ${ }^{1 a}$

\begin{tabular}{lccc}
\hline & bR & ppR & $\Delta \mathrm{E}_{\mathrm{ppR}-\mathrm{bR}}$ \\
\hline \hline protein $^{b}$ & 2.65 & 3.00 & 0.35 \\
HBN model $^{c}$ & 2.91 & 3.01 & 0.10
\end{tabular}

${ }^{a}$ From QM calculations on the chromophore. ${ }^{b} \overline{\text { External point charges included in QM }}$ calculations. ${ }^{c}$ Only point charges of Asp85/75, Asp212/201, Arg82/85, W402, W401 and W406/400 included in QM calculation.

Hence, the increased distance between the Schiff base nitrogen and Asp212 in bR relative to ppR (5.2 $\AA$ in bR, $4.5 \AA$ in ppR) in their model do not influence the spectral shift to the extent they concluded.

(1) Hayashi, S.; Tajkhorshid, E.; Pebay-Peyroula, E.; Royant, A.; Landau, E. M.; J., N.; Schulten, K. J. Phys. Chem. B 2001, 105, 10124-10131.

(2) Ren, L.; Martin, C. H.; Wise, K. J.; Gillespie, N. B.; Luecke, H.; Lanyi, J. K.; Spudich, J. L.; Birge, R. R. Biochemistry 2001, 40, 13906-13914.

(3) Böttcher, C. J. F. Theory of Electric Polarization.; volume 1 Elsevier Scientific Publ. Co.: Amsterdam, 2 ed.; 1973.

(4) This approximation, common in atomic polarization models, describes a medium which can be separated into linear polarizable centers ${ }^{3}$.

(5) $\Delta_{S_{1}-S_{0}}$ denotes the difference of a contribution, e.g., the electrostatic interaction energy $V_{\text {ch-pr }}^{\text {ES }}$, between the $\mathrm{S}_{1}$ and the $\mathrm{S}_{0}$ state. 